\title{
MANEJO DE PACIENTES GERIÁTRICOS TRANSGÊNEROS
}

Alexandre Ricardo Farret Junior ${ }^{1}$, Cristian Koch Weber ${ }^{1}$, Mariana Menegon de Souza ${ }^{1}$, Leonardo Bosi Moireira*1, Miriam Heine ${ }^{2}$

\section{Resumo}

INTRODUÇÃO: A disforia de gênero (DG) é uma condição na qual o indivíduo não se identifica com o gênero de nascimento biológico [1]. Houve uma inclusão e disponibilidade do tratamento para DG no Sistema Único de Saúde desde 2008, aumentando a população total de indivíduos atendidos no Brasil [2]. OBJETIVO: Revisar informações para manejo clínico do paciente idoso transgênero ou em terapia de redesignação de sexo. MÉTODO: Revisão sistemática de 298 artigos encontrados no Pubmed através da estratégia PICO, sendo incluidos 8 artigos para compor as referências. Os critérios de inclusão foram disponibilidade de acesso ao artigo, estudos com população de transgêneros idosos e estudos com relevância para prática clínica. RESULTADOS: O manejo do paciente idoso transgênero deve ser abordado nos seguintes aspectos: cuidado psicossocial, buscando-se trabalhar a isolação, a vergonha, a ausência de suporte [3] e a perspectiva em relação ao envelhecimento e ao processo de redesignação de gênero [4]. Além disso, é irrefutável garantir atendimento respeitoso não-binário devido aos históricos de abuso e discriminação nos sistemas de saúde [5]; cuidado hormonal, pois as perdas hormonais são naturais no processo de senilidade e intensificam a DG, sendo a perda de testosterona um fator de risco para depressão e doenças cardiovasculares. $\mathrm{O}$ uso de reposição hormonal nesse grupo de pacientes deve ser intensamente monitorado, pois o metabolismo dos idosos tende a ser mais lentificado, apresentando-se como um desafio na prática clínica [6]; cuidado especial, pois há estudos que associam o abuso de hormônios com desenvolvimento de câncer de próstata [7], prolactinoma [8]. CONCLUSÃO: O manejo de pacientes geriátricos transgêneros é um desafio duplo para a prática clínica. A equipe de saúde enfrenta o processo natural de envelhecimento somado à 
disforia de gênero. É preciso ressaltar que ainda não há preparo suficiente para lidar com esse grupo na ausência de uma equipe multidisciplinar e de centros de referência, que envolvam aspectos biopsicossociais do paciente e de sua rede de apoio. O profissional deve se informar sobre as particularidades dos pacientes, incluindo conhecimento sobre a reposição hormonal. De tal maneira, caminha-se, respeitosamente, para uma melhor qualidade de vida dessa minoria.

Palavras Chave: transgênero; geriatria; disforia; gênero.

1 Acadêmicos de medicina da Universidade Luterana do Brasil, Canoas/RS.

2 Docente do curso de medicina da Universidade Luterana do Brasil, Canoas/RS.

* Apresentador do trabalho 


\section{Referências}

GÓMEZ-GIL, E.; ZUBIAURRE-ELORZA, L.; DE ANTONIO, IE.; GUILLAMON, A.; SALAMERO, M. Determinants of quality of life in Spanish transsexuals attending a gender unit before genital sex reassignment surgery. In: Qual Life Res. Espanha, 2013. 23:671-678.

PORTAL BRASIL. Cirurgias de mudança de sexo são realizadas pelo SUS desde 2008. Disponível em: < http://www.brasil.gov.br/cidadania-ejustica/2015/03/cirurgias-de-mudanca-de-sexo-sao-realizadas-pelo-sus-desde2008>. Acesso em 20 de março de 2016.

ETTNER, R.; WYLIE, K. Psychological and social adjustment in older transsexual people. In: Maturitas. Irlanda, 2012. 74: 226-229.

FABBRE, VD. Gender Transitions in Later Life: The Significance of Time in Queer Aging. In: J Gerontol Soc Work. USA, 2014. 57: 161-175.

TORKE, A. American Geriatrics Society Care of Lesbian, Gay, Bisexual, and Transgender Older Adults Position Statement. In: American Geriatrics Society Ethics Committee. USA, 2015. 63: 423-426.

ETTNER, R. Care of the elderly transgender patient. In: Curr Opin Endocrinol Diabetes Obes. USA, 2013. 20: 580-584.

GOOREN, L.; MORGENTALER, A. Prostate cancer incidence in orchidectomised male-to-female transsexual persons treated with oestrogens. In: First International Journal of Andrology. USA, 2014. 46: 1156-1160.

CUNHA, FS.; DOMENICE, S.; CÂMARA, VL.; SIRCILI, MHP.; GOOREN, LJG.; MENDONÇA, BB.; COSTA, EMF. Diagnosis of prolactinoma in two male-tofemale transsexual subjects following high-dose cross-sex hormone therapy. In: First International Journal of Andrology. USA, 2014. 47: 680-684. 